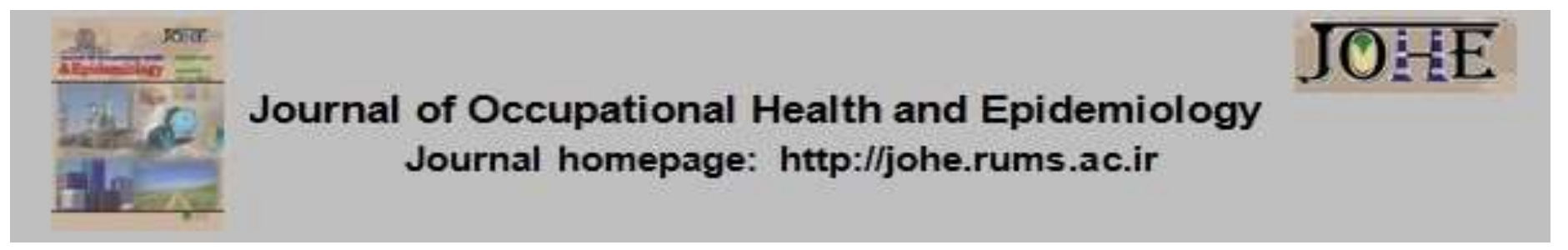

\title{
Factors associated with malignant skin tumors among patients referred to Ali-ibn Abi Talib Hospital, Rafsanjan, Iran, (2011-2013)
}

\author{
Tahereh Eslammanesh $^{1 *}$, Zahra Mohit ${ }^{2}$, Mohsen Rezaeian ${ }^{3}$, Sina Amoozgar ${ }^{4}$ \\ 1- Assistant Prof., Social Determeinants of Health Research Center, Rafsanjan University of Medical Sciences, Rafsanjan, Iran. \\ 2- Dermatologist, Rafsanjan University of Medical Sciences, Rafsanjan, Iran. \\ 3- Professor, Social Determeinants of Health Research Center, Rafsanjan University of Medical Sciences, Rafsanjan, Iran. \\ 4- Medical Student, Rafsanjan University of Medical Sciences, Rafsanjan, Iran.
}

\section{Citation: Eslammanesh T, Mohit Z, Rezaeian M, Amoozgar S. Factors associated with
malignant skin tumors among patients referred to Ali-ibn Abi Talib Hospital, Rafsanjan, Iran,
$(2011-2013)$. JOHE. 2018; 7(3):139-44.}

Article Info

\section{* Corresponding authors:}

Tahereh Eslammanesh,

E-mail:

dr.eslammanesh@yahoo.com

\section{Article history}

Received: Oct, 2016

Accepted: Jul, 2018

10.29252/johe.7.3.139

Print ISSN: 2251-8096 Online ISSN: 2252-0902

Peer review under
responsibility of Journal of
Occupational Health and
Epidemiology

\section{Abstract}

Background: Malignant skin tumors are of the most common types of cancers worldwide. Rafsanjan County, Iran, is geographically located in a warm and arid area. In addition, due to farming activity, a large population of this county is exposed to sunlight. The present study aimed at to determine factors associated with malignant skin tumors among patients referred to Ali-ibn Abi Talib Hospital, Rafsanjan, Iran, (2011-2013).

Materials and Methods: In this cross-sectional study, medical records and histologic slides of patients referring to the pathology department of Ali-ebn Abi Taleb hospital in Rafsanjan during the years 2011-2013, with a definitive diagnosis of malignant skin tumors, were extracted. The slides were reviewed to determine the subtypes of each tumor type. A researcher-made checklist including demographic characteristics, tumor type, and subtypes was completed, and the data were analyzed using descriptive statistics.

Results: Of 70 patients, 69 patients had a single tumor and 1 patient had 2 skin lesions. There were 71 cases of malignant skin tumors, including $87.3 \%, 9.9 \%$, and $2.8 \%$ cases of basal cell carcinoma (BCC), squamous cell carcinoma (SCC), and melanoma, respectively. The ratio of men to women in total skin tumors was 1.7 to $1.48 .6 \%$ of patients consisted of farmers. The most frequent cites for BCC and SCC were head and neck with frequency of $96.8 \%$ and $85.7 \%$, respectively, and for melanoma, lower limb with frequency of $100 \%$, was the most frequent cite.

Conclusion: The results of this study indicated that skin cancer was more common among older men and farmers. It is recommended that training and screening programs be carried out to reduce risk factors, and prevent and detect these tumor types, especially among at-risk groups.

Keywords: Basal Cell Carcinoma, Squamous Cell Carcinoma, Melanoma, Iran.

\section{Introduction}

Malignant skin tumors are divided into two groups of non-melanoma skin cancers and malignant melanoma. Non-melanoma skin cancers, including basal cell carcinoma (BCC) and squamous cell carcinoma (SCC), are the most common malignancies among the white race (1). In people with light skin color, BCC and SCC account for approximately $75-80$ and $20-25$ percent of nonmelanoma skin cancers, respectively. Since the incidence of non-melanoma skin cancers has an increasing trend, this neoplasm is an important issue in health care (2).

SCC is more common among white people, and is often created in the head, neck, and upper limbs (1). In SCC, the incidence ratio of men to women is 3 to 1 , and its prevalence increases after the age of 60 
years. The mortality rate is higher among white and elderly people, and the incidence of deaths due to SCC among men is 3 times that of women (1).

Ultraviolet (UV) radiation is the main risk factor for SCC, so that the incidence of SCC is directly related to exposure to sunlight. Other risk factors for SCC include various skin scars like burn scars, X-ray, chronic osteomyelitis sinuses, and hidradenitis suppurativa. Contact with chemicals such as arsenic, coal, and soot is also a risk factor. The histologic subtypes of SCC include adenoid SCC, spindle cell SCC, verrucous SCC, mucin producing SCC, and basaloid SCC (3).

$\mathrm{BCC}$ is the most common human malignancy, and accounts for almost $50 \%$ of cases. This tumor mostly occurs in white people over the age of 40 years. The upper two thirds of the face is the most common site of involvement. This area is chronically exposed to sunlight (4). However, BCC is seen in areas protected from sunlight, and sites of chronic venous stasis, arteriovenous malformation (AVM), arsenic ingestion, $\mathrm{X}$-ray exposure, chickenpox scars, tattoos, and hair transplantation scars (3). The most common subtype is the nodular-ulcerative tumor followed by the superficial subtype, which is more common in the trunk (5).

Most melanomas are associated with exposure to sunlight and UV radiation; hence, it is often seen in the head and neck and lower extremities. White and blond people and people who experience freckles or its aggravation after exposure to sunlight, and redheads are prone to melanoma. Individuals with a large number of melanocytes are also at risk of melanoma (3). The most common histological subtypes of the tumor are superficial spreading, nodular, lentigo maligna, and acral lentiginous melanoma (6).

The county of Rafsanjan, Iran, is located on the southern edge of the Lut Desert with a population of about 300 thousand people and at an altitude of $1460 \mathrm{~m}$ above sea level. The average precipitation in this city is $100 \mathrm{~mm}$, and it is one of the largest pistachio producing regions in the world (7).

In Rafsanjan County, due to its location on the margin of the Lut Desert and the large number of its residents employed in agriculture, and hence, their exposure to pesticides, the rate of skin tumors seems to be high. Therefore, the researchers in this study decided to perform a retrospective crosssectional study on patient files and a review of the frequency of skin tumors among patients referring to the pathology department of Ali-ibn Abi Talib hospital in Rafsanjan.

\section{Materials and Methods}

The sample of this cross-sectional study included pathology records and slides of patients with skin tumors referring to the pathology department of Aliibn Abi Talib hospital in Rafsanjan $(n=70)$ between the years 2011 and 2013. The diagnosis of pathology, age, sex, occupation, location, and number of lesions were extracted from the pathology records present in the pathology department's archives.

In cases in which the occupation of patients was not mentioned in the application form, information was obtained by phone call to patients. Individuals who had incomplete records and could not be contacted were excluded from the study.

Moreover, the pathology slides of all patients were extracted from the archives of the department, and used by the pathologist executing the plan for revising the histological profile and determining the subtype of each type of tumor.

The data were collected and coded, then, analyzed in SPSS software (version 18.0, SPSS Inc., Chicago, IL, USA) using descriptive statistics including rate (\%) for qualitative variables and mean \pm standard deviation (SD) for quantitative variables.

\section{Results}

In this study, 70 patients were enrolled. However, 1 person had two types of tumors; therefore, 71 tumors were examined. The mean \pm SD of subjects' age was $67.20 \pm 12.08$ years (in the range of 36 to 96 years); in addition, $26(37.1 \%)$ and $44(62.9 \%)$ of the subjects were men and women, respectively. The highest rate was related to farmers with $48.6 \%$, followed by housewives (34.3\%), construction workers $(7.1 \%)$, teachers $(5.7 \%)$, and livestock farmers $(2.9 \%)$; the lowest rate $(1.4 \%)$ was associated with welders (Figure 1).

Of these 71 tumors, 62 (87.3\%), 7(9.9\%), and $2(2.8 \%)$ cases were BCC, SCC, and melanoma, respectively. The investigation of the frequency distribution of malignant skin tumors by year indicated that the highest and lowest rates of BCC tumors were observed in 2012 (43.5\%) and 2013 $(25.8 \%)$, respectively. Of the two cases of melanoma, one took place in 2012, and the other in 2013. In addition, the highest rate of SCC $(57.1 \%)$ was related to 2012 . 


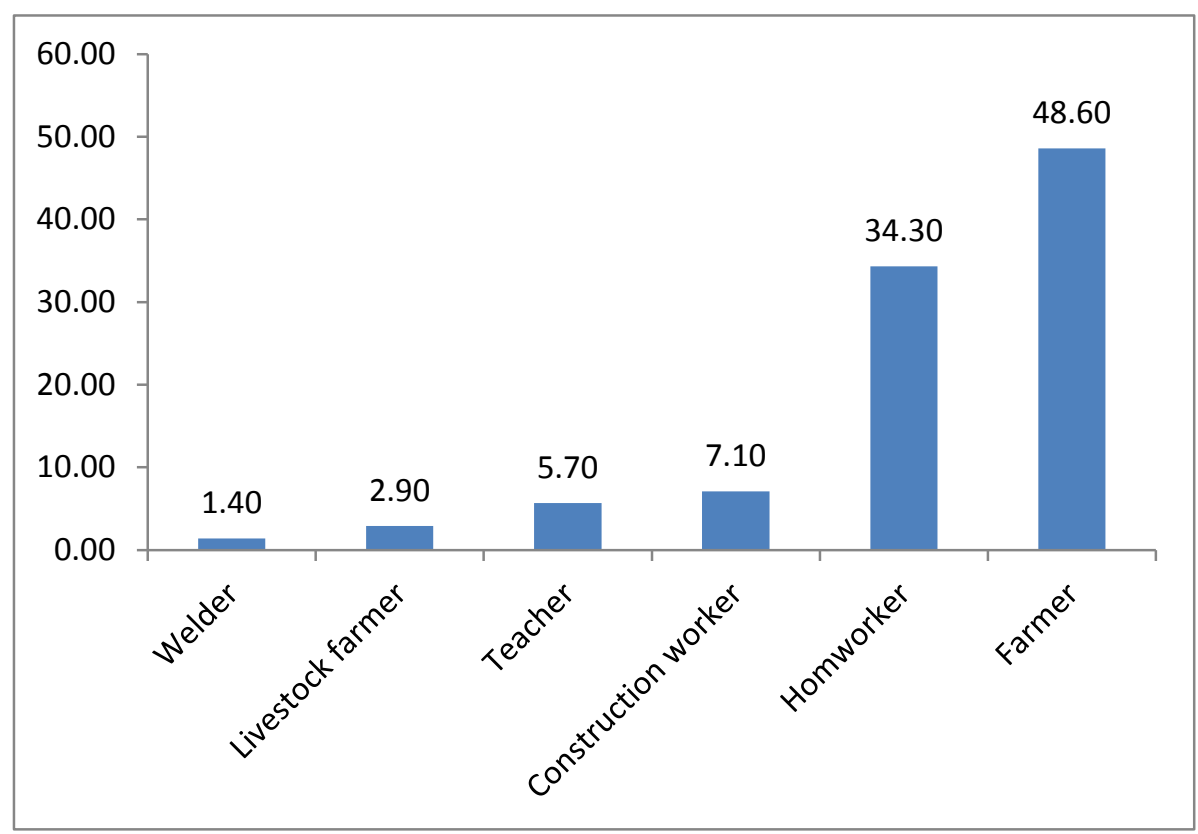

Figure 1: Rate of skin tumors by occupation

Most cases of BCC (48.4\%) and SCC (71.4\%) were observed among farmers. Of the two melanomatype tumors, one was seen in a man farmer and one in a housewife. Moreover, 93.0\%, 4.2\%, 1.4\%, and $1.4 \%$ of the skin tumors were in the head and neck area, lower extremities, upper limb, and the trunk, respectively (Figure 2 ).

In addition, $62.9 \%$ and $37.1 \%$ of BCC cases were observed among men and women, respectively. Most cases of SCC (71.4\%) were seen among men and $28.6 \%$ was observed among women. The incidence of melanoma was equal $(50 \%)$ in both genders.

Investigation of the rate of BCC tumor subtypes indicated that the most common subtype was nodular with a rate of $46.8 \%$ followed by adenoid with a rate of $11.3 \%$. The rates of nodular adenoid, pigmented adenoid, and morphea-like nodular were
$8.1 \%, 4.8 \%$, and $4.8 \%$, respectively. In addition, examining the rate of BCC subtypes in terms of the anatomical position indicated that the nodular subtype with $45.0 \%$ was the most common subtype of $\mathrm{BCC}$ in the head and neck region. The results showed that the prevalence of adenoid and nodular subtypes was higher among men (71.4\%). Moreover, adenoid was more common in farmers with the rate of $57.1 \%$. There were four subtypes of morphea-like, which were seen in combination with other subtypes. All four subtypes were present among man farmers.

The rates of SCC subtypes of type grade II, usual type grade I, and basaloid were $57.1 \%, 28.6 \%$, and $14.3 \%$, respectively. The prevalence of SCC subtypes indicated that the usual type grade II with a rate of $66.7 \%$ was the most common SCC subtype in the head and neck region. One case of basaloid was also observed in the groin region.

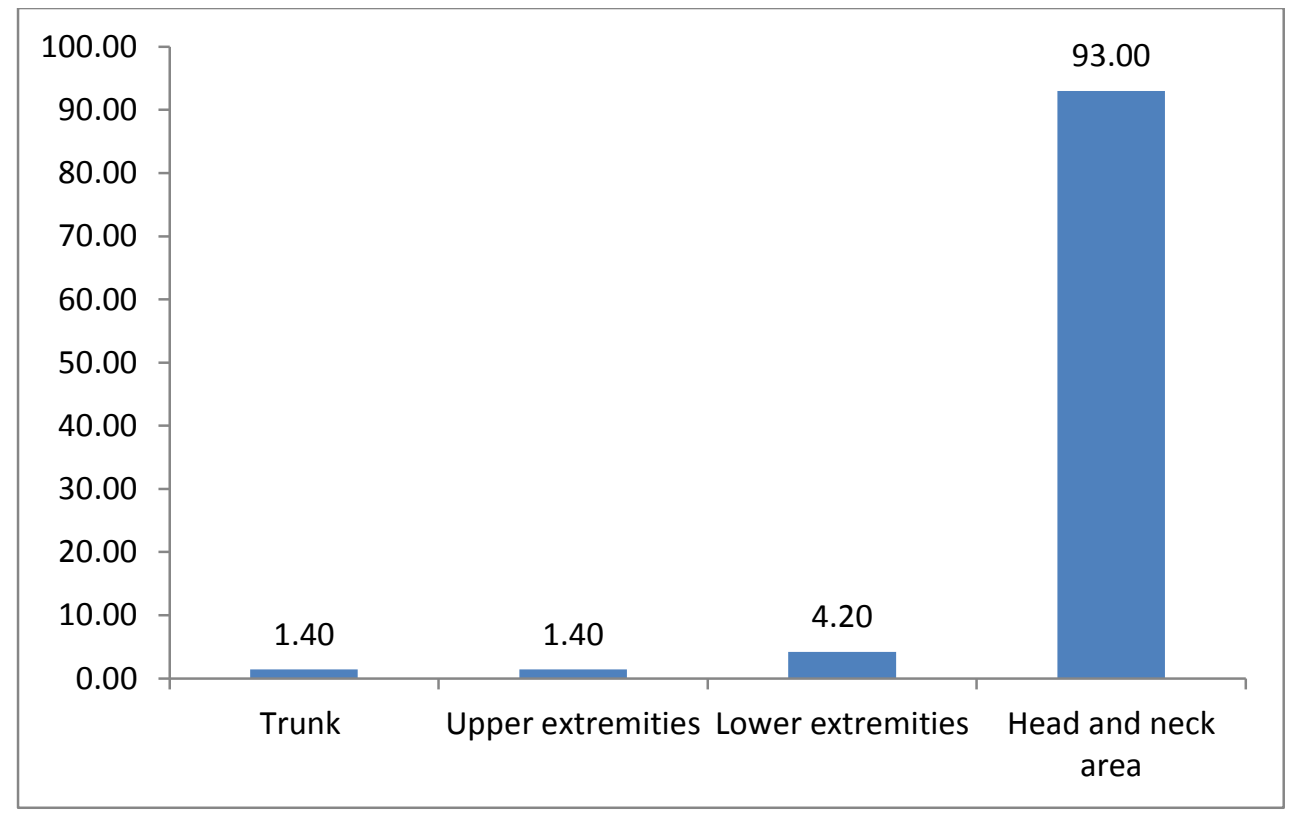

Figure 2: Rate of skin tumors by anatomical position 
Evaluation of the rate of SCC subtypes in terms of sex showed that there were 3 and 1 cases of usual type grade II among men and women, respectively. Furthermore, there were 2 cases of usual type grade I and 1 case of basaloid subtype among men and women, respectively. The most prevalent subtype of SCC was also usual type grade II, that 3 and 1 cases of which were, respectively, observed among farmers and a homemaker.

In this study, one patient suffered from both SCC and BCC. The SCC of the patient was grade II with BCC superficial type on the margin of the lesion. The site of this lesion was on the nose, and this tumor was observed in a 63-year-old man farmer.

Of the 71 tumors examined, 2 cases of melanoma were diagnosed, both belonging to the nodular subtype, 1 in a man and 1 in a woman. In addition, one of them was a construction worker and the other was a housewife. In terms of anatomical distribution, both cases were observed in the lower extremities (one in the leg and the other in the heel).

\section{Discussion}

In the present study, the BCC, SCC, and melanoma incidence rates were $87.3 \%, 9.9 \%$, and $2.8 \%$, respectively. Yazdanfar and Ghasemi estimated the incidence of BCC and SCC as, respectively, 59\% and $29 \%$ in Hamadan Province, Iran (8). Moreover, Golchai et al. reported the incidence rates of BCC and SCC in Guilan Province, Iran, as 64\% and 28\%, respectively (9).

The mean \pm SD of the age of subjects was $67.20 \pm$ 12.48 years. In the study by Yazdanfar and Ghasemi, the mean age of the patients was 61.6 years (8). The peak age for BCC and melanoma in the study by Lotfinejad et al. was 60-69 years, and it was reported to be 70-79 years for SCC (10). Other studies have also shown that the incidence of malignant skin tumors was higher in older ages (11, 12). In this respect, the results of this study are in line with those of the present study.

Moreover, of the patients with skin tumors in this study, $62.9 \%$ and $37.1 \%$ were men and women, respectively. This rate was $62.9 \%$ and $37.1 \%$, $56.8 \%$ and $43.2 \%$, and $67.2 \%$ and $38.8 \%$ in the studies by Lotfinejad et al. (10), Khakzad (13), and Yazdanfar and Ghasemi (8), respectively. Dorak and Karpuzoglu (14) and Staples et al. (15) also achieved similar results. In this regard, the results of the present study are consistent with their studies.

In the present study, the highest rate of malignant skin tumors was observed among farmers followed by homemakers with $48.6 \%$ and $34.3 \%$, respectively. In the study by Yazdanfar and Ghasemi, the highest occupational rate was related to workers $(38.0 \%)$ followed by homemakers
$(31.1 \%)$, respectively. In this study, the meaning of the worker was not accurately defined; however, according to the dominant culture, it was most probably the construction workers (8). This rate was first related to farmers $(28.20 \%)$, and then, homemaker $(25.66 \%)$ in the study conducted by Lotfinejad et al. (10). In this regard, the results of this study were consistent with the abovementioned studies. Since nearly one-third of skin tumors are reported among women and most of these individuals are homemakers, it can be concluded that housekeeping ranks second among occupations. Farmers receive the highest amount of sunlight due to the nature of their occupation; hence, it is logical for this group to rank first in skin tumors.

In this study, the most prevalent type of tumor was BCC with a rate of $87.3 \%$ followed by SCC $(9.9 \%)$, and finally, melanoma (2.8\%). In the study performed by Valavi et al. in Khuzestan Province, Iran, BCC was reported among $70.4 \%$ of patients, and other cases included SCC, malignant melanoma, and other cancers with rates of $14.1 \%$, $3.7 \%$, and $11.8 \%$, respectively (16). In a study conducted in Mashhad City, Iran, the incidence rates of BCC, SCC, malignant melanoma, and metastasis to the skin were $57.7 \%, 20.2 \%, 4.4 \%$, and $4.4 \%$, respectively (17). The incidence of BCC in this study was higher than that of all of the abovementioned studies.

In the present study, melanoma accounted for only $2(2.8 \%)$ of the 71 tumors under investigation. However, the incidence rates of this type of skin tumor were $4.90 \%, 4.64 \%$, and $4.50 \%$ in the studies conducted by Golchai et al. in Guilan Province between 1991 and 1998 (9), Lotfinejad et al. in Urmia Province during a 10-year period from 1991 to 2001 (10), and Yazdanfar and Ghasemi in Hamadan Province during a 15-year period from 1991 to 2007 (8), respectively. This can be due to the short time period and fewer patients examined in the current study. In the present study, both cases of melanoma had occurred in the lower extremities. In the study by Lotfinejad et al., 23 out of 51 cases of melanoma were found in the upper and lower extremities (10).

In the present study, $93.0 \%$ of skin tumors were observed in the head and neck region. Khakzad also reported the head and neck region as the most common site of skin tumors (13). In a study on patients with SCC in Kerman, Iran, the head and neck region was the incidence site of SCC in $77 \%$ of cases (18). In a study in Mashhad, $93 \%$ of BCC tumors and $74 \%$ of SCC cases were observed in the head and neck region, and lower extremities were the most common site for melanoma (17). Since the face is the most exposed region to radiation from 
the sun or other sources, the head and neck area is more prone to skin tumors compared to other parts of the body. In a study on 62 patients with malignant melanoma in Khuzestan, Iran, most cases of disease were observed in the lower limb (16); however, in the study by Revenga et al. in Spain, melanoma was more likely to be found in the trunk and lower extremities among men and women, respectively. This is due to the fact that these areas are more likely to be exposed to acute and intermittent UV radiation; hence, they are at a greater risk of sunburn and the appearance of melanoma (19).

In the present study, the most common subtype of BCC tumors was nodular with a rate of $46.8 \%$. This rate varied in different studies. For example, in the studies by Toosi et al. (20), Stoica et al. (21), and Chang and Gao (22), the prevalence of the nodular subtype was $68 \%, 44.95 \%$, and $53.9 \%$, respectively. However, in the study by Robinson et al., the nodular subtype had a prevalence of $54 \%$ (23). In addition, Koyuncuer reported a prevalence of $47.6 \%$ for the nodular subtype (24).

The present study also had some limitations. In the completion of the checklist, some patients did not cooperate well, and did not respond appropriately to the questions; for instance, the variable of exposure to sunlight was eliminated for this reason. In addition, the relocation of patients to hospitals of nearby cities and the lack of access to the histopathology results of skin tumors of individuals visiting specialized centers challenged the access to real statistics. Therefore, it is suggested that a similar prospective study be fulfilled in the future, and for a longer period of time; so that all the necessary issues can be considered.

\section{Conclusion}

The results of this study indicate that skin cancer is more common among men compared to women, and most of the cases are observed among the elderly and farmers. This group is often at constant and intense exposure to sunlight; so it is recommended that adequate training be provided for individuals in the community, especially those working in the sun, to prevent the harmful effects of sunlight.

\section{Acknowledgement}

The contributions made by all those participating in the study in one way or other are appreciated.

Conflict of interest: None declared.

\section{References}

1. Bolognia JL, Jorizzo JL, Schaffer JV.Dermatology. $3^{\text {rd }}$ ed, Philadelphia, United States: Saunders; 2012.

2. Jemal A, Siegel R, Ward E, Hao Y, Xu J, Murray T, et al. Cancer statistics, 2008. CA Cancer J Clin 2008; 58(2):71-96.

3. Rosai J. Rosai and Ackerman's surgical pathology. 10th ed. Maryland Heights, Missouri, United States: Mosby; 2011. Vol 1. P.134-60.

4. Ferreira FR, Pevide Bda C, Rodrigues RF, Nascimento LF, Lira ML.Differences in age and topographic distribution of the different histological subtypes of basal cell carcinoma, Taubaté (SP), Brazil. An Bras Dermatol 2013; 88(5):726-30.

5. Junior WB, Di Chiacchio N, Criado PR. Tratado de dermatologia. 2nd ed. Sao Paulo, Brazil: Atheneu; 2010. Vol 2.

6. Reed JA, Shea CR. Lentigo maligna: melanoma in situ on chronically sun-damaged skin. Arch Pathol Lab Med 2011; 135(7):838-41.

7. Rafsanjan city. [Internet]. 2018 July 20. Available from:https://en.rums.ac.ir/index.aspx?fkeyid=\&siteid= 63\&pageid $=7719$

8. Yazdanfar A, Ghasemi E. Frequency of skin cancers in Hamedan from 1991 to 2007. Journal of Dermatology and Cosmetic 2011; 2(2):115-23.

9. Golchai J, Sobhani AR, Feyzi Khah MR, Fathi CH. Survey of malignant skin tumors in Guilan. Journal of Guilan University of Medical Sciences 2002; 11(42):16.

10. Lotfinejad Sh, Rashidi T, Eshghi M. Prevalence of malignant skin tumors among patients referring to Urmia health centers, 1991-2001. Journal of Ardabil University of Medical Sciences 2003; 3(4):33-8.

11. Jacobsen AA, Galvan A, Lachapelle CC, Wohl CB, Kirsner RS, Strasswimmer J. Defining the need for skin cancer prevention education in uninsured, minority, and immigrant communities. JAMA Dermatol2016; 152(12):1342-7.

12. Merrill SJ, Subramanian M, GodarDE. Worldwide cutaneous malignant melanoma incidences analyzed by sex, age, and skin type over time (1955-2007): Is HPV infection of androgenic hair follicular melanocytes a risk factor for developing melanoma exclusively in people of European-ancestry? Dermatoendocrinol 2016; 8(1):e1215391.

13. Khakzad M. Skin cancers in patients referred for plastic surgery. Journal of Babol University of Medical Sciences 2003; 5(1):50-4.

14. Dorak MT, Karpuzoglu E. Gender differences in cancer susceptibility: an inadequately addressed issue. Front Genet 2012; 3:268.

15. Staples M, Marks R, Giles G. Trends in the incidence of non-melanocytic skin cancer (NMSC) treated in Australia 1985-1995: are primary prevention programs starting to have an effect? Int $\mathrm{J}$ Cancer 1998;78(2):144-8

16. Valavi E, Rafie Sh, Pakseresht $P$, Siadat S.Prevalence of skin cancer in southwest of Iran. Koomesh 2013; 15(1):83-8.

17. Amouzgar MH, Yazdanpanah MJ, Ebrahimirad M. Frequency of different skin cancer in Qaem hospital, Mashhad, from 1975-1995: A cross-sectional study. Iranian Journal of Dermatology 2006; 9(1):28-34.

18. Rahnama Z, Haghdoost AA. Relationship of sex, age and site of the skin squamous cell carcinoma with pathological grading. Iranian Journal of Dermatology 2002; 6(21):10-15. 
19. Revenga Arranz F, Paricio Rubio JF, Mar Vazquez Salvado M, del Villar Sordo V. Descriptive epidemiology of basal cell carcinoma and cutaneous squamous cell carcinoma in Soria (north-eastern Spain) 1998-2000: a hospital-based survey. JEur Acad Dermatol Venereol 2004; 18(2):137-41.

20. Toosi P, Sami Kermani S, Shirzadian Kebria A. Epidemiology of malignant cutaneous tumors in Loghman, Hakim and Bouali hospitals, 2001-2002. Tehran University Medical Journal 2004; 62(6):50917.

21. Stoica LE, Georgescu CV, Pătraşcu V, Radu CC, Tolea I, Mogoantă L. Basal cell carcinomas - clinical- evolutional and histopahotologic aspects. Curr Health Sci J 2009; 35(4):228-33.

22. Chang JM, Gao XM. Clinical and histopathological characteristics of basal cell carcinoma in Chinese patients. Chin Med J (Engl) 2013; 126(2):211-4.

23. Robinson AJ, Walsh M, Hill C. Histopathological variation of incompletely excised basal cell carcinoma's and the variation with the grade of surgeon - implications for revalidation. Eur J Surg Oncol 2015; 41(1):165-8.

24. Koyuncuer A. Histopathological evaluation of nonmelanoma skin cancer. World JSurg Oncol 2014; 12:159. 